\title{
Droplet resonator based optofluidic microlasers
}

\author{
Alper Kiraz ${ }^{a}$, Alexandr Jonáś ${ }^{b}$, Mehdi Aas $^{a}$, Yasin Karadag ${ }^{a}$, Oto Brzobohatýc, Jan Ježek ${ }^{c}$, \\ Zdeněk Pilát ${ }^{c}$, Pavel Zemánek ${ }^{c}$, Suman Anand ${ }^{d}$, and David McGloin ${ }^{d}$ \\ ${ }^{a}$ Department of Physics, Koç University, Rumelifeneri Yolu, Sariyer, 34450 Istanbul, Turkey; \\ ${ }^{b}$ Department of Physics Engineering, Istanbul Technical University, 34469 Maslak, Istanbul, \\ Turkey; \\ ${ }^{c}$ Institute of Scientific Instruments of the ASCR, v.v.i., Královopolská 147, 61264 Brno, Czech \\ Republic; \\ ${ }^{d}$ Electronic Engineering and Physics Division, University of Dundee, Nethergate, Dundee DD1 \\ $4 \mathrm{HN}, \mathrm{UK}$
}

\begin{abstract}
We introduce tunable optofluidic microlasers based on active optical resonant cavities formed by optically stretched, dye-doped emulsion droplets confined in a dual-beam optical trap. To achieve tunable dye lasing, optically pumped droplets of oil dispersed in water are stretched by light in the dual-beam trap. Subsequently, resonant path lengths of whispering gallery modes (WGMs) propagating in the droplet are modified, leading to shifts in the microlaser emission wavelengths. We also report lasing in airborne, Rhodamine B-doped glycerolwater droplets which were localized using optical tweezers. While being trapped near the focal point of an infrared laser, the droplets were pumped with a Q-switched green laser. Furthermore, biological lasing in droplets supported by a superhydrophobic surface is demonstrated using a solution of Venus variant of the yellow fluorescent protein or E. Coli bacterial cells expressing stably the Venus protein. Our results may lead to new ways of probing airborne particles, exploiting the high sensitivity of stimulated emission to small perturbations in the droplet laser cavity and the gain medium.
\end{abstract}

Keywords: microdroplet laser, whispering gallery mode, optical tweezers, optical stretcher, optical manipulation, dye laser, biological laser

\section{INTRODUCTION}

Optofluidic sources of laser light that can be integrated into lab-on-a-chip systems allow for dynamic control of laser resonator geometry and gain medium and, open up new paradigms in bio-sensing. ${ }^{1}$ With their unique features, liquid droplets stand out among different optical resonator alternatives for developing optofluidic lasers. Thanks to their spherical geometry and smooth surface, droplet-based cavities host high quality optical resonances called whispering gallery modes (WGMs). ${ }^{2}$ These low-loss resonant modes then allow droplet lasers to operate at low threshold pump powers. Liquid droplets are easy to produce using aerosol generators in air or using flow focusing or T-junction geometries in a microfluidic chip. Upon generation, droplets can be captured and manipulated using optical micromanipulation techniques such as optical tweezing or stretching. Their easily deformable nature makes them attractive for developing tunable optical components, e.g. tunable light sources. In addition, water-based droplet cavities are also biologically compatible, allowing for the use of aqueous solutions of biologically relevant molecules as laser gain media.

Here, we demonstrate dye lasing in optically manipulated emulsion droplets surrounded by another liquid or liquid aerosol droplets surrounded by air. We were also able to tune the lasing WGMs of emulsion droplets by optical stretching in a dual beam optical trap. Finally, we demonstrate lasing in droplet resonators containing biological gain media such as fluorescent proteins or fluorescent protein expressing bacterial cells. These results can pave the way for new paradigms in biological sensing with droplet resonator lasers.

Further author information: (Send correspondence to A.K.)

A.K.: E-mail: akiraz@ku.edu.tr, Telephone: +902123381701

Laser Resonators, Microresonators, and Beam Control XVI, edited by Alexis V. Kudryashov, Alan H. Paxton, Vladimir S. Ilchenko, Lutz Aschke, Kunihiko Washio, Proc. of SPIE

Vol. 8960, 896015 - (c) 2014 SPIE · CCC code: 0277-786X/14/\$18 - doi: 10.1117/12.2041447

Proc. of SPIE Vol. $8960896015-1$ 


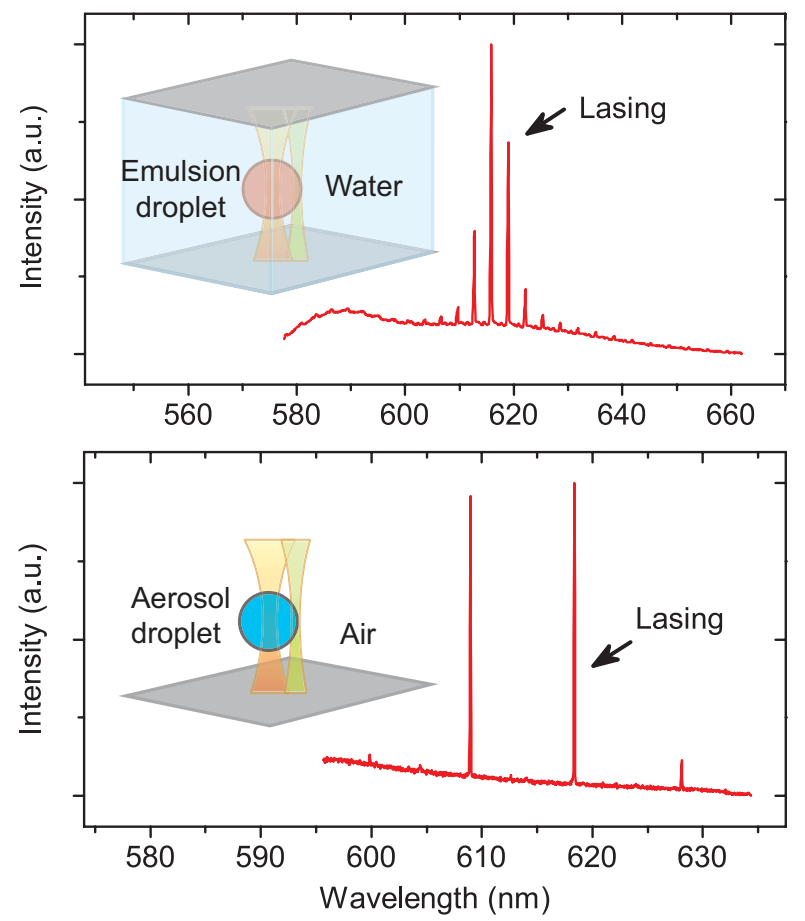

Figure 1. Geometry and spectrua of dye doped lasing liquid microdroplets optically trapped and manipulated in 3D using a high numerical aperture microscope objective, $\mathrm{NA}=1.2$, and excited with a $532 \mathrm{~nm}$ Q-switched pulsed laser, a) schematic of an oil emulsion microdroplet manipulated inside a chamber made of two microscope cover glass filled with water b) WGM lasing spectrum of an immersion oil microdroplet with $27 \mu \mathrm{m}$ diameter and $n_{o}=1.515$ refractive index, doped with Dil(3) (1,1-Dioctadecyl-3,3,3 ,3-tetramethylindo carbocyanine perchlorate) inside water with refractive index of $n_{w}=1.334$, c) schematic of an aerosol microdroplet dispensed from a piezo electric nebulizer and trapped above a plasma treated hydrophilic microscope cover glass, d) WGM lasing spectrum of an aerosol microdroplet with $9 \mu$ m diameter made of $39 \mathrm{wt} \%$ glycerol/water with refractive index of 1.38 doped with 1mM Rhodamine B.

\section{DROPLET RESONATOR LASERS MANIPULATED BY OPTICAL TWEEZERS}

Lasing in airborne microdroplets was first successfully demonstrated almost thirty years ago in freely flowing streams of droplets. ${ }^{3}$ However, freely moving liquid aerosols have only a limited use in practical applications in sensing and microlaser design. In order to fully exploit the potential of lasing WGMs in microdroplets, it is necessary to stabilize the droplet position over extended time periods. The position stabilization of droplets of water and other polar liquids can be effectively realized by depositing them on a superhydrophobic surface. ${ }^{4}$ Active, non-invasive control of the droplet position by external micromanipulation then represents the next step in the practical exploitation of lasing aerosols. To date, manipulation of lasing airborne microdroplets has been demonstrated in electrodynamic ${ }^{5}$ and acoustic ${ }^{6}$ trapping configurations.

Optical tweezing is another micromanipulation technique that is especially well suited for stable confinement of very small droplets and readily adaptable for simultaneous manipulation of large numbers of particles. ${ }^{7}$ Various studies employed optical tweezing to localize liquid aerosols over long periods of time for applications in a large variety of fields including atmospheric chemistry and physics, and health science. ${ }^{8,9}$ Using an experimental setup that combined independent continuous trapping laser beam at $1064 \mathrm{~nm}$ and pulsed pump laser beam at $532 \mathrm{~nm}$ at the focus of a high numerical aperture microscope objective, we were able to optically pump droplets that were simultaneously manipulated in three dimensions (Fig. 1). Thanks to the dye gain medium placed in the droplets, dye lasing was observed for two experimental configurations: glycerol/water droplets manipulated in air ${ }^{10}$ and immersion oil droplets manipulated in water. ${ }^{11}$ In both cases, tens of laser emission spectra could be recorded from the trapped droplets before photobleaching of the dye contained in the droplets. 


\section{TUNABLE DROPLET RESONATOR LASERS BY OPTICAL STRETCHING}

Tunability of shape, size, and composition is an important advantage of reconfigurable optofluidic components based on microdroplets. The most straightforward way of tuning spectral position of WGMs of airborne droplets relies on controlled changes of the liquid cavity size using evaporation/condensation, ${ }^{12}$ electrowetting, ${ }^{13}$ or photothermal effect. ${ }^{14}$ Adjustment of resonant frequency of microdroplet optical cavity can also be achieved by controlled deformation of the microdroplet which lifts the degeneracy of WGMs with different azimuthal mode numbers. Frequency splitting of the azimuthal WGMs then depends on the extent of the microdroplet deformation. ${ }^{15,16}$ For microdroplets surrounded by a solid elastomer, tuning has been achieved by mechanical shape deformation ${ }^{17}$ or by changing refractive index of liquid crystal droplets in external electric field. ${ }^{18}$ However, for the case of microdroplets surrounded by another liquid in microfluidic channels, reversible spectral tuning has not been shown yet, despite different demonstrations of optical functionality of emulsion droplets including dye lasing. ${ }^{?, 19}$ In order to address the issue of spectral tuning, researchers have demonstrated multicolor dye lasing in microfluidic channels by fast switching between consecutive droplets doped with different dye molecules. ${ }^{20}$ Recently, emission wavelength tuning by continuous decrease of the droplet size due to dissolving of benzyl alcohol microdroplets in water has also been demonstrated. ${ }^{21}$ Nevertheless, such dissolution-based tuning of the lasing emission wavelength is irreversible.

As shown by Guck et al., transparent dielectric microobjects suspended in a liquid can be deformed by the forces of light. ${ }^{22}$ Optically induced deformation of soft microobjects (lipid vesicles, living cells) has been successfully demonstrated using a pair of counter-propagating laser beams in the so-called optical stretcher geometry. ${ }^{22}$ Optical stretcher represents a convenient, all-optical method for tuning the emission spectra of lasing microdroplets via controlled droplet deformation that leads to frequency-splitting of degenerate WGMs of originally spherical droplets. However, the magnitude of optical stretching forces is rather small in comparison with the typical interfacial tension forces acting between two immiscible liquids in emulsions. In order to achieve sufficient droplet deformations without using prohibitively high stretching laser powers, interfacial tension has to be lowered by several orders of magnitude. This can be accomplished by using various mixtures of surfactant molecules and inorganic salts for the emulsion preparation ${ }^{23}$ and Ward et al. ${ }^{24}$ in the single beam and multiple beam optical tweezers, respectively, to observe large deformations of the emulsion droplets with moderate trapping powers. Despite the above demonstrations, optical stretcher geometry has not been employed yet for light-induced tuning of the emission spectra of lasing microdroplets.

We introduce novel tunable optofluidic dye microlasers that use optical stretching forces for the adjustment of the laser emission wavelength. ${ }^{25}$ Our microlasers are based on droplets of immersion oil emulsified in water and confined in a dual-beam optical trap. Since the droplets are stained with a fluorescent dye, they function as active ultrahigh-Q optical resonant cavities that are optically pumped with an independent pulsed green laser. In order to reduce the stretching power required for microlaser tuning, we lower the interfacial tension between the droplets and aqueous host medium to $\sim 1 \mathrm{mN} / \mathrm{m}$ using a surfactant and sodium chloride. In order to excite different WGMs in the stretched droplets, the pump beam was focused at different locations on the droplet: (i) The droplet rim in the polar plane containing the dual beam trap axis (on-axis excitation in Fig. 2), (ii) the droplet the rim in the equatorial plane perpendicular to the dual beam trap axis (off-axis excitation in Fig 2), and (iii) the droplet center (on-center excitation in Fig. 2). Tuning slopes of $0.49 \mathrm{~nm} / \mathrm{W}$ and $-0.52 \mathrm{~nm} / \mathrm{W}$ were observed for on-axis and off-axis excitation cases, respectively. While, for on-center excitation all WGMs with positive and negative tuning slopes were simultaneously excited. These results were well explained by simulations when changes of the droplet size and refractive index of both the droplet and the host medium resulting from the droplet heating by the stretching laser beam are also taken into consideration.

\section{DROPLET BASED BIOLOGICAL LASERS}

Bio-inspired photonic devices rely on the use of biological species as the components of a photonic device. In this field, the most recognized recent developments have been the demonstrations of living lasers using GFPexpressing mammalian ${ }^{26}$ or E. Coli bacterial $^{27}$ cells as the gain media placed inside a Fabry-Perot resonator and in-vitro lasing from fluorescent proteins using a ring resonator. ${ }^{28}$ In our experiments we employed resonators based on aqueous droplets standing on a superhydrophobic surface. ${ }^{12}$ The studied droplets contained laser gain medium in the form of a solution of Venus variant of the yellow fluorescent protein (Fig. 3) or E. Coli bacterial 


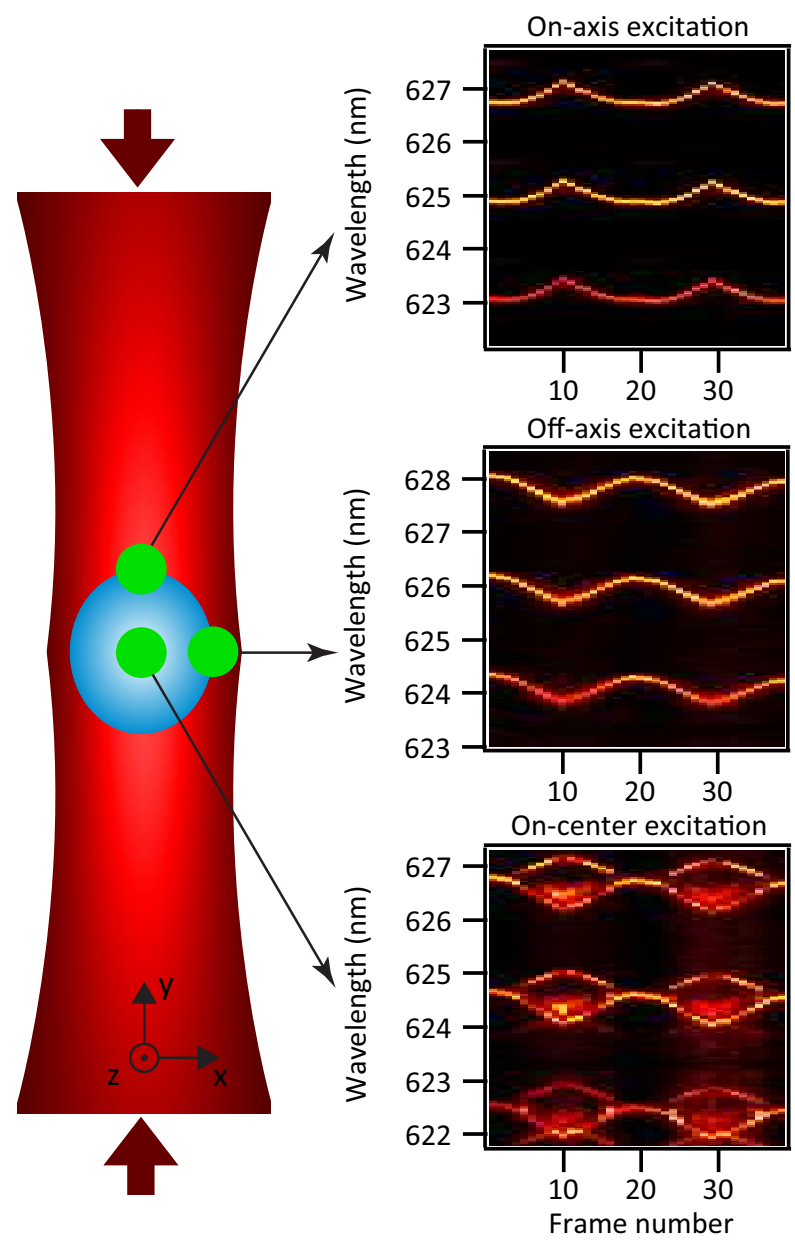

Figure 2. Spectral tuning of a lasing microdroplet by optical stretching in a dual-beam optical trap.

cells expressing stably the Venus protein (Fig. 4). Upon optical pumping with femtosecond laser pulses at $488 \mathrm{~nm}$, Venus lasing was observed in both cases. For the case of aqueous solution of Venus fluorescent protein, stable laser emission was observed while with bacterial cells, fluctuations were observed in the laser emission intensity due to diffusion/active motion of single bacterial cells in and out of the excitation volume.

\section{CONCLUSION}

In summary, optofluidic microlasers with droplet resonators can serve as tunable light sources that can be optically manipulated and employed e.g. for local probing. They can also be used for sensitive chemical and biological analysis because of the high sensitivity of stimulated emission to small perturbations in the shape, size, and material properties of the droplet cavity. Further potential for bio-sensing based on droplet lasers lies in exploring FRET phenomenon. ${ }^{28}$ Finally, aqueous droplet microlasers are compatible with biological gain media. Such biological microlasers can be self-healing, i.e. they allow for the regeneration of the gain medium based on fluorescent proteins following photobleaching. Self-recognition and self-assembly of biological molecules can be exploited to program and modulated the laser characteristics, thus paving the way to new ways of bio-sensing.

\section{ACKNOWLEDGMENTS}

The authors acknowledge support from TÜBİTAK (Grants No. 111T059and 112T972), Czech Science Foundation (P205/11/P294), Technology Agency of the Czech Republic (TA03010642), Ministry of Education, Youth and 

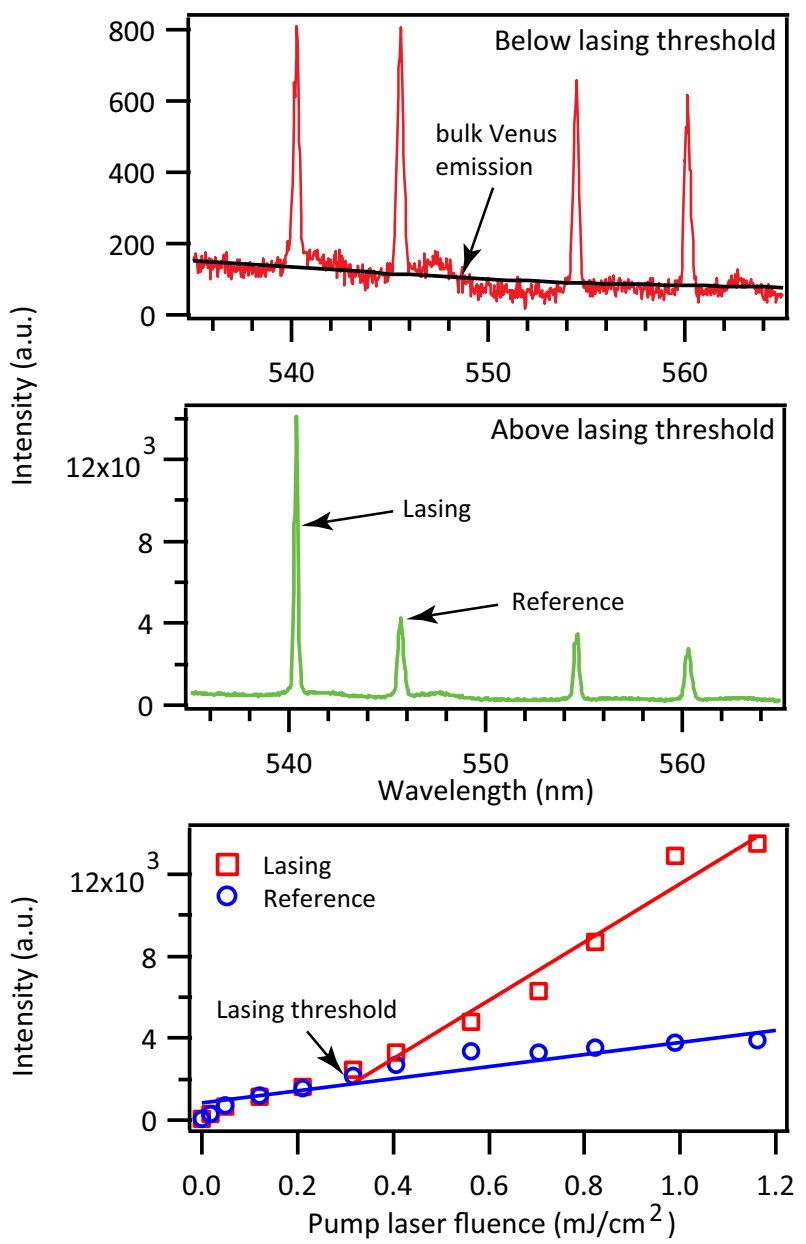

Figure 3. Lasing in a microdroplet doped with a solution of the Venus variant of the yellow fluorescent protein.

Sports of the Czech Republic together with the European Commission (ALISI CZ.1.05/2.1.00/01.0017). The authors thank Martin Šiler for help with thermal analysis and A. L. Demirel for help with the surface tension measurements.

\section{REFERENCES}

[1] Li, Z. and Psaltis, D., "Optofluidic dye lasers," Microfluid. Nanofluid. 4, 145-158 (2008).

[2] Ashkin, A. and Dziedzic, J. M., "Observation of resonances in the radiation pressure on dielectric spheres," Phys. Rev. Lett. 38, 1351 (1977).

[3] Tzeng, H.-M., Wall, K. F., Long, M. B., and Chang, R. K., "Laser emission from individual droplets at wavelengths corresponding to morphology-dependent resonances," Opt. Lett. 9, 499-501 (1984).

[4] Kiraz, A., Sennaroglu, A., Doganay, S., Dundar, M. A., Kurt, A., Kalaycoglu, H., and Demirel, A. L., "Lasing from single, stationary, dye-doped glycerol/water microdroplets located on a superhydrophobic surface," Opt. Commun. 276, 145-148 (2007).

[5] Tona, M. and Kimura, M., "Novel lasing modes observed in a levitated single dye-doped microdroplet," Journal of the Physical Society of Japan 69, 3533-3535 (2000).

[6] Azzouz, H., Alkhafadiji, L., Balslev, S., Johansson, J., Mortensen, N. A., Nilsson, S., and Kristensen, A., "Levitated droplet dye laser," Opt. Express 14, 4374-4379 (2006). 


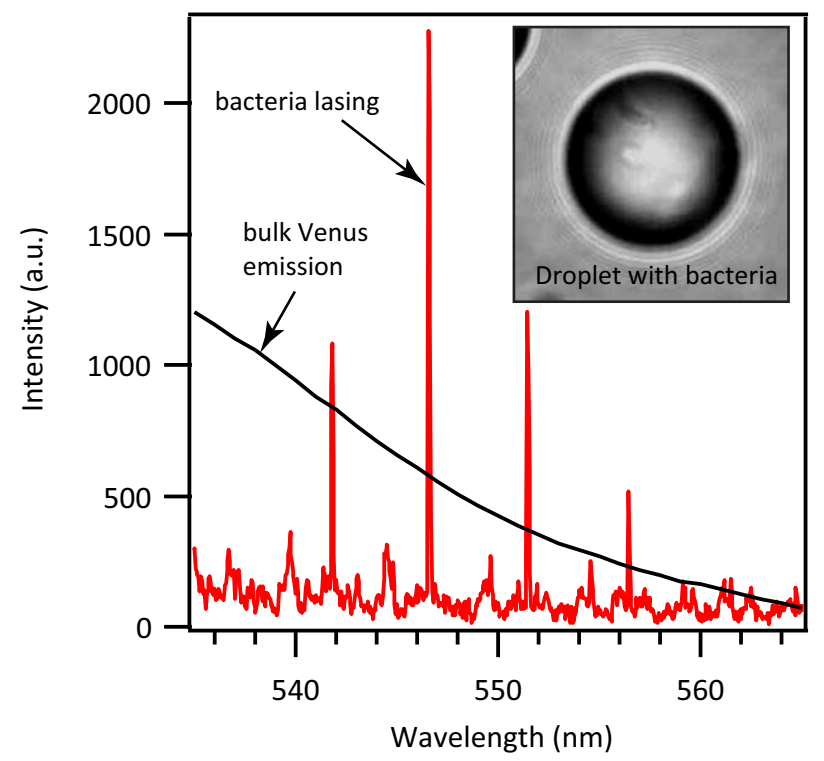

Figure 4. Lasing in a microdroplet containing E. Coli bacterial cells expressing stably the Venus protein.

[7] Burnham, D. R. and McGloin, D., "Holographic optical trapping of aerosol droplets," Optics Express 14, 4176-4182 (2006).

[8] Hopkins, R. J., Mitchem, L., Ward, A. D., and Reid, J. P., "Control and characterisation of a single aerosol droplet in a single-beam gradient-force optical trap," Phys. Chem. Chem. Phys. 6, 4924 (2004).

[9] McGloin, D., Burnham, D. R., Summers, M. D., Rudd, D., Dewar, N., and Anand, S., "Optical manipulation of airborne particles: techniques and applications," Faraday Discuss. 137, 335-350 (2008).

[10] Karadag, Y., Aas, M., Jonáš, A., Anand, S., McGloin, D., and Kiraz, A., "Dye lasing in optically manipulated liquid aerosols," Opt. Lett. 38(10), 1669-1671 (2013).

[11] Aas, M., Jonáš, A., and Kiraz, A., "Lasing in optically manipulated, dye-doped emulsion microdroplets," Opt. Commun. 290, 183-187 (2013).

[12] Kiraz, A., Kurt, A., Dündar, M. A., and Demirel, A. L., "Simple largely tunable optical microcavity," Appl. Phys. Lett. 89, 081118 (2006).

[13] Kiraz, A., Karadă̆, Y., and Coskun, A. F., "Spectral tuning of liquid microdroplets standing on a superhydrophobic surface using electrowetting," Appl. Phys. Lett. 92, 191104 (2008).

[14] Kiraz, A., Karadag, Y., Yorulmaz, S. C., and Muradoglu, M., "Reversible photothermal tuning of a salty water microdroplet," Phys. Chem. Chem. Phys. 11, 2597-2560 (2009).

[15] Chen, G., Mazumder, M. M., Chemla, Y. R., Serpengüzel, A., Chang, R. K., and Hill, S. C., "Wavelength variation of laser emission along the entire rim of slightly deformed microdroplets," Opt. Lett. 18, 1993-1995 (1993).

[16] Yorulmaz, S. C., Mestre, M., Muradoglu, M., Alaca, B. E., and Kiraz, A., "Controlled observation of nondegenerate cavity modes in a microdroplet on a superhydrophobic surface," Opt. Commun. 282, 30243027 (2009).

[17] Saito, M., Shimatani, H., and Naruhashi, H., "Tunable whispering gallery mode emission from a microdroplet in elastomer," Optics Express 16, 11915-11919 (2008).

[18] Humar, M., Ravnik, M., Pajk, S., and Muševič, I., "Electrically tunable liquid crystal optical microresonators," Nature Photonics 3, 595-600 (2009).

[19] Tanyeri, M., Perron, R., and Kennedy, I. M., "Lasing droplets in a microfabricated channel," Optics Letters 32, 2529-2531 (2007).

[20] Tang, S. K. Y., Li, Z., Abate, A. R., Agresti, J. J., Weitz, D. A., Psaltisd, D., and Whitesides, G. M., "A multi-color fast-switching microfluidic droplet dye laser," Lab on a Chip 9, 2767-2771 (2009). 
[21] Tang, S. K. Y., Derda, R., Quan, Q., Loncar, M., and Whitesides, G. M., "Continuously tunable microdroplet-laser in a microfluidic channel," Opt. Express 19, 2204-2215 (2011).

[22] Guck, J., Ananthakrishnan, R., Mahmood, H., Moon, T. J., Cunningham, C. C., and Käs, J., "The optical stretcher: A novel laser tool to micromanipulate cells," Biophys. J. 81, 767-784 (2001).

[23] Møller, P. C. F. and Oddershede, L. B., "Quantification of droplet deformation by electromagnetic trapping," Europhys. Lett. 88, 48005 (2009).

[24] Ward, A. D., Berry, M. G., Mellorb, C. D., and Bain, C. D., "Optical sculpture: controlled deformation of emulsion droplets with ultralow interfacial tensions using optical tweezers," Chemical Communications 43, 4515-4517 (2006).

[25] Aas, M., Jonáš, A., Kiraz, A., Brzobohatý, O., Ježek, J., Pilát, Z., and Zemánek, P., "Spectral tuning of lasing emission from optofluidic droplet microlasers using optical stretching," Opt. Express 21(18), 2138021394 (2013).

[26] Gather, M. C. and Yun, S. H., "Single cell biological lasers," Nature Photonics 5, 406-410 (2011).

[27] Gather, M. C. and Yun, S. H., "Lasing from escherichia coli bacteria genetically programmed to express green fluorescent protein," Opt. Lett. 36, 3299-3301 (2011).

[28] Chen, Q., Zhang, X., Sun, Y., Ritt, M., Sivaramakrishnan, S., and Fan, X., "Highly sensitive fluorescent protein fret detection using optofluidic lasers," Lab on a Chip 13, 2679-2681 (2013). 